\title{
Influence of the interaction between Ac-SDKP and Ang II on the pathogenesis and development of silicotic fibrosis
}

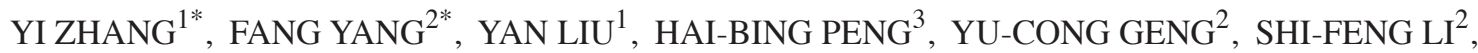 \\ HONG XU ${ }^{2}$, LI-YAN ZHU ${ }^{1}$, XIU-HONG YANG ${ }^{1}$ and DARRELL BRANN ${ }^{4}$ \\ ${ }^{1}$ Basic Medical College, ${ }^{2}$ Medical Research Center and ${ }^{3} \mathrm{Ji}$ Tang College, North China University of Science and Technology, \\ Tangshan, Hebei 063210, P.R. China; ${ }^{4}$ Department of Neuroscience and Regenerative Medicine, \\ Medical College of Georgia, Augusta University, Augusta, GA 30912, USA
}

Received August 21, 2017; Accepted December 18, 2017

DOI: $10.3892 / \mathrm{mmr} .2018 .8824$

\begin{abstract}
N-acetyl-seryl-aspartyl-lysyl-proline (Ac-SDKP) is a natural tetrapeptide that is released from thymosin $\beta 4$ by prolyl oligopeptides. It is hydrolyzed by the key enzyme of the renin-angiotensin system, angiotensin-converting enzyme (ACE). The aim of the present study was to investigate the alterations in Ac-SDKP and the ACE/angiotensin II (Ang II)/angiotensin II type 1 (AT1) receptor axis and its impact on the pathogenesis and development of silicotic fibrosis. For in vivo studies, a HOPE MED 8050 exposure control apparatus was used to establish different stages of silicosis in a rat model treated with Ac-SDKP. For in vitro studies, cultured primary lung fibroblasts were induced to differentiate into myofibroblasts by Ang II, and were pretreated with Ac-SDKP and valsartan. The results of the present study revealed that, during silicosis development, ACE/Ang II/AT1 expression in local lung tissues increased, whereas that of Ac-SDKP decreased. Ac-SDKP and the ACE/AT1/Ang II axis were inversely altered in the development of silicotic fibrosis. Ac-SDKP treatment had an anti-fibrotic effect in vivo. Compared with the silicosis group, the expression of $\alpha$-smooth muscle actin ( $\alpha$-SMA), Collagen (Col) I, Fibronectin (Fn) and AT1 were significantly downregulated, whereas matrix metalloproteinase-1 (MMP-1)
\end{abstract}

Correspondence to: Dr Yan Liu, Basic Medical College, North China University of Science and Technology, 21 Bohai Avenue, Caofeidian Xincheng, Tangshan, Hebei 063210, P.R. China

E-mail: liuyan.zone@163.com

*Contributed equally

Abbreviations: Ac-SDKP, N-acetyl-seryl-aspartyl-lysyl-proline; ECM, extracellular matrix; ACE, angiotensin-converting enzyme; Ang II, angiotensin II; RAS, renin-angiotensin system; $\alpha$-SMA, $\alpha$-smooth muscle actin; TGF- $\beta$, transforming growth factor- $\beta$; H\&E, hematoxylin and eosin; IHC, immunohistochemistry; MMP-1, matrix metalloproteinase-1; TIMP-1, tissue inhibitor of metalloproteinases-1

Key words: N-acetyl-seryl-aspartyl-lysyl-proline, silicosis, reninangiotensin system, myofibroblast expression and the MMP-1/tissue inhibitor of metalloproteinases-1 (TIMP-1) ratio was increased in the Ac-SDKP treatment group. In vitro, pre-treatment with Ac-SDKP or valsartan attenuated the expression of $\alpha$-SMA, Col I, Fn and AT1 in Ang II-induced fibroblasts. In addition, MMP-1 expression and the MMP-1/TIMP-1 ratio were significantly higher in Ac-SDKP and valsartan pre-treatment groups compared with the Ang II group. In conclusion, the results of the present study suggest that an imbalance between Ac-SDKP and ACE/Ang II/AT1 molecules promotes the development of silicosis and that Ac-SDKP protects against silicotic fibrosis by inhibiting Ang II-induced myofibroblast differentiation and extracellular matrix production.

\section{Introduction}

Silicosis is caused by long-term inhalation of silicon dioxide dust in professional activities and its basic pathological features include the formation of silicotic nodules and lung fibrosis (1). $\mathrm{N}$-acetyl-seryl-aspartyl-lysyl-proline (Ac-SDKP) is a natural tetrapeptide formed from its precursor, thymosin $\beta 4$, released by prolyl oligopeptides (2). Ac-SDKP has antifibrosis effects and is expressed in human and animal tissues $(3,4)$. The downregulation of Ac-SDKP expression is associated with organ fibrosis $(5,6)$. The renin-angiotensin system (RAS) is a key mediator of lung fibrosis $(7,8)$; the key enzyme of RAS, angiotensin-converting enzyme (ACE), consists of 2 catalytic domains, namely the $\mathrm{C}$ and $\mathrm{N}$ domains (9). The $\mathrm{C}$ domain catalyzes angiotensin (Ang) I to form Ang II (10). Ang II is the major active protein of RAS and its fibrotic effect is primarily mediated by angiotensin II type 1 (AT1), which includes the induction of inflammatory responses as well as increased expression of transforming growth factor- $\beta$ (TGF- $\beta$ ), proliferation of fibroblasts and extracellular matrix (ECM) deposition (11-14). Ac-SDKP is cleared by the $\mathrm{N}$ domain of $\mathrm{ACE}$ and its plasma and tissue levels therefore significantly increase following ACE inhibition $(3,15)$. The role of ACE in producing the pro-fibrotic molecule Ang II and degrading the anti-fibrotic molecule Ac-SDKP suggests that the interaction between Ac-SDKP and RAS may be important in silicosis. In the present study, changes in Ac-SDKP and Ang II signaling during the development of silicosis were investigated. The 
protective effect of Ac-SDKP and its potential association with Ang II signals was also investigated.

\section{Materials and methods}

Materials. A total of 100 specific pathogen-free male Wistar rats (3 weeks of age) were obtained from the Vital River Laboratory (Beijing, China). The rats were housed in a temperature-controlled facility at $22 \pm 2^{\circ} \mathrm{C}, 50 \pm 10 \%$ humidity and $0.03-0.04 \% \mathrm{CO}_{2}$, with a $12 \mathrm{~h}$ light/dark cycle and received food and water according to the guidelines established by the North China University of Science and Technology (16). HOPE MED 8050 exposure control apparatus was supplied by Tianjin Hope Industry and Trade Co., Ltd. (Tianjin, China). $\mathrm{SiO}_{2}$ was obtained from Sigma Aldrich (Merck KGaA, Darmstadt, Germany). The silica content of $\mathrm{SiO}_{2}$ was $>99 \%$ and its dust particle size distribution was as follows: $97 \%<5 \mu \mathrm{m}$ diameter, $80 \%<3 \mu \mathrm{m}$ diameter (according to the supplier). Ac-SDKP was supplied by Bachem (Bubendorf, Switzerland). Ang II and valsartan were purchased from Sigma-Aldrich (Merck KGaA). Ac-SDKP (cat. no. CSB-E0925r) and Ang II (cat. no. CSB-E04494r) ELISA kits were obtained from CUSABIO (Wuhan, Hubei, China). The PV-6000 1-step polymer detection system for mouse, rabbit and rat antibodies were acquired from Beijing Zhongshan Jinqiao Biotechnology Co., Ltd. (Beijing, China). Enhanced chemiluminescence reagent (cat. no. RPN2232) was supplied by GE Healthcare (Chicago, IL, USA). Masson's trichrome stain was supplied by Baso Diagnostics, Inc. (Wuhan, China). Polyclonal rabbit anti-collagen (Col) I (cat. no. ab34710), anti $\alpha$-smooth muscle actin (SMA; cat. no. ab32575), anti-Fibronectin (Fn) (cat. no. ab45688), rabbit monoclonal anti-AT1 (cat. no. ab124734) and mouse monoclonal anti-ACE (cat. no. ab117334) were purchased from Abcam (Cambridge, UK). Polyclonal rabbit anti-matrix metalloproteinase (MMP)-1 (cat. no. EL800296-50) and anti-tissue inhibitor of metalloproteinases-1 (TIMP-1; cat. no. EL800628-50) were obtained from Eterlife, Ltd. (Birmingham, UK). Polyclonal mouse anti- $\beta$-actin (cat. no. sc-47778) and polyclonal rabbit anti-GAPDH (cat. no. sc-25778) were purchased from Santa Cruz Biotechnology, Inc. (Dallas, TX, USA). Dulbecco's modified Eagle's medium (DMEM) was obtained from Biological Industries USA (Cromwell, CT, USA). Fetal bovine serum (FBS) was purchased from Gibco (Thermo Fisher Scientific, Inc., Waltham, MA, USA).

Animal studies. All animal studies were approved by the Institutional Animal Care and Use Committee of North China University of Science and Technology. HOPE MED 8050 exposure control apparatus was used to create the silicosis model. The settings were as follows: Cabinet temperature, $20-25^{\circ} \mathrm{C}$; humidity, $70-75 \%$; pressure, -50 to $+50 \mathrm{~Pa}$; oxygen concentration, 20\%; flow rate of $\mathrm{SiO}_{2}: 3.0-3.5 \mathrm{~m} / \mathrm{min}$; dust mass concentration, $2,000 \mathrm{mg} / \mathrm{m}^{3}$. Each rat inhaled $\mathrm{SiO}_{2}$ for $3 \mathrm{~h}$ per day for $0,2,4,8,12$ and 16 weeks. The rats were randomly divided into 6 groups $(\mathrm{n}=10)$ and inhaled dust for $0,2,4,8,12$ and 16 weeks to observe dynamic changes during the development of silicosis. To determine the anti-silicotic effect of Ac-SDKP, rats were randomly divided into 3 groups $(n=10)$ : i) 16 week control (treated with $0.9 \%$ saline for
16 weeks); ii) 16 week silicosis (treated with $0.9 \%$ saline $48 \mathrm{~h}$ before $\mathrm{SiO}_{2}$ inhalation and thereafter for 16 weeks); and iii) Ac-SDKP post-treatment (inhaled $\mathrm{SiO}_{2}$ and then treated with $0.9 \%$ saline for 8 weeks and Ac-SDKP for another 8 weeks). Ac-SDKP ( $800 \mathrm{mg} / \mathrm{kg} /$ day) or control ( $0.9 \%$ saline) was administered via a mini-osmotic pump implanted into the abdominal cavity as previously described (17). The rats were anesthetized, and sera and lung samples were collected and frozen at $-80^{\circ} \mathrm{C}$ until subsequent analysis.

Cell culture. Rats $(\mathrm{n}=10)$ were anaesthetized and lung tissue was removed. Lung fibroblasts were isolated from minced tissue, as previously described (18) and plated on $25-\mathrm{cm}^{2}$ plates containing DMEM supplemented with $10 \%$ FBS and $1 \%$ penicillin-streptomycin. The cells were cultured in a humidified atmosphere containing $5 \% \mathrm{CO}_{2}$ at $37^{\circ} \mathrm{C}$. Cells at $80 \%$ confluence were cultured in FBS-free DMEM for $24 \mathrm{~h}$, when most cells were quiescent. The induction effect of Ang II $\left(10^{-7} \mathrm{~mol} / \mathrm{l}\right)$ on the transformation of lung fibroblasts into myofibroblasts was studied at different time points $(0,5,15$ and $30 \mathrm{~min}$, and $1,3,6,12,24$ and $48 \mathrm{~h}$ ) at $37^{\circ} \mathrm{C}$. Primary lung fibroblasts were divided into four groups and cultured for $24 \mathrm{~h}$ at $37^{\circ} \mathrm{C}$ : i) Control (FBS-free DMEM); ii) Ang II $\left(10^{-7} \mathrm{~mol} / \mathrm{l}\right)$; iii) Ang II + Ac-SDKP ( $\left.10^{-8} \mathrm{~mol} / \mathrm{l}\right)$; and iv) Ang II + valsartan $\left(10^{-6} \mathrm{~mol} / \mathrm{l}\right)$.

Lung histopathology. The right lower lungs were fixed in $4 \%$ paraformaldehyde at $4^{\circ} \mathrm{C}$ for $24 \mathrm{~h}$, embedded in paraffin, sectioned at $5 \mu \mathrm{m}$ and subjected to hematoxylin and eosin (H\&E) and Masson trichrome staining at room temperature for pathophysiological observation. For H\&E stainng, the tissues sections were stained with hematoxylin for $30 \mathrm{sec}$ and with stain $1 \%$ eosin solution for $1 \mathrm{~min}$. For Masson trichrome staining, the tissue sections were stained with Weigert's working hematoxylin for $10 \mathrm{~min}$. Subsequently, the sections were washed in running tap water for $5 \mathrm{~min}$. Biebrich scarlet staining was subsequently performed for $5 \mathrm{~min}$. Phosphomolybdic acid was added for $10 \mathrm{~min}$ and then the solution was discarded. Tissue samples were transferred directly into Aniline blue for $5 \mathrm{~min}$ and rinsed in distilled water. Images in each rat were obtained from 5 randomly selected fields of view using Olympus DP80 light microscope (magnification, x200; Olympus Corporation, Tokyo, Japan).

Immunohistochemistry (IHC). IHC of lung tissue sections $(5 \mu \mathrm{m})$ and cultured fibroblasts (passage 9) was performed. Paraffin embedded sections were deparaffinized, rehydrated and underwent removal of endogenous peroxidase with $3 \% \mathrm{H}_{2} \mathrm{O}_{2}$ and antigen retrieval was performed using $0.01 \mathrm{M}$ citrate buffer. Following blocking with $5 \%$ bovine serum albumin (Sigma-Aldrich, Merck KGaA) in $0.1 \mathrm{M}$ phosphate-buffer saline (PBS) for $30 \mathrm{~min}$ at room temperature to reduce nonspecific binding, samples were incubated with a primary antibody against $\alpha$-SMA (1:200; cat. no. ab32575; Abcam) overnight at $4^{\circ} \mathrm{C}$, followed by incubation with a goat anti-mouse/rabbit secondary antibody (cat. no. PV-6000, ready to use solution; OriGene Technologies, Inc., Beijing, China) at $37^{\circ} \mathrm{C}$ for $1 \mathrm{~h}$. Immunoreactivity was visualized with 5\% 3,3'-diaminobenzidene (cat. no. ZLI-9018; OriGene Technologies, Inc., Beijing, China) at room temperature for 

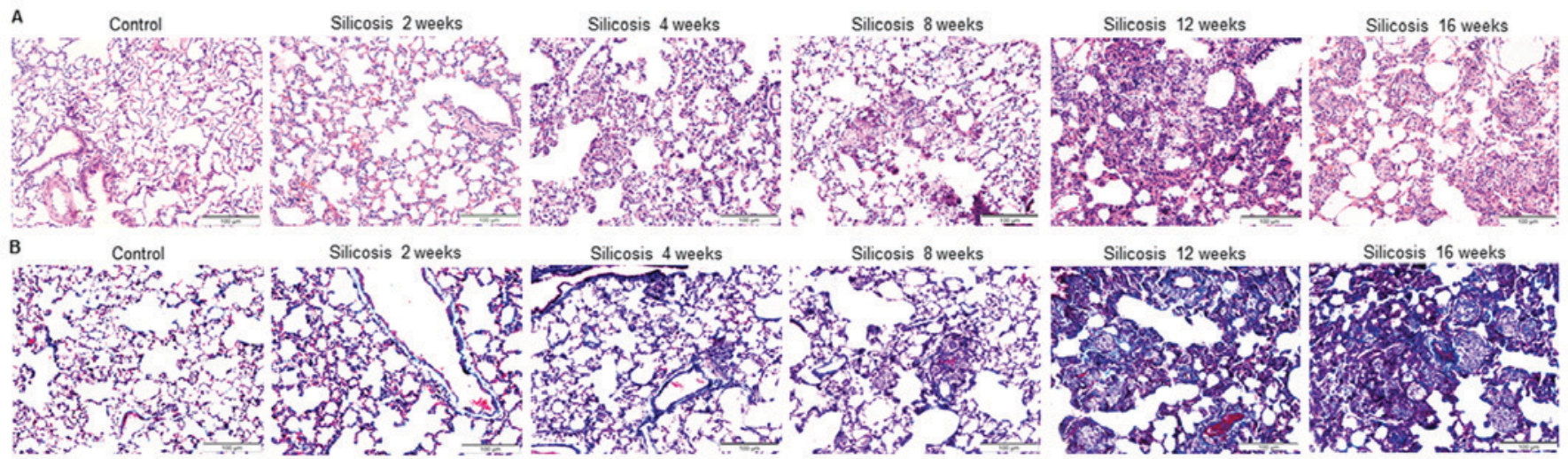

Silicosis 12 weeks

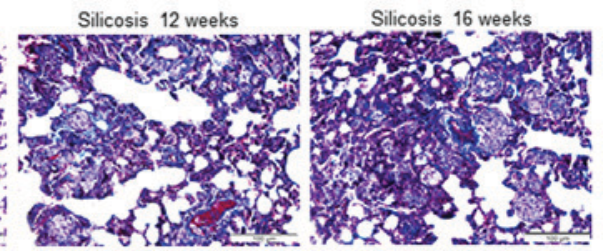

Figure 1. Histomorphological evaluation of the lung tissues. (A) Hematoxylin and eosin and (B) Masson stained lung tissues. Scale bars, $100 \mu \mathrm{m}$.

5 min. Brown staining was considered positive. PBS, instead of primary antibody, was used as a negative control and $\alpha$-SMA staining of vascular smooth muscle cells was used as a positive control. Images were obtained from 5 separate randomly selected fields of view using the Olympus DP80 (magnification, $\mathrm{x} 200$ ).

Western blotting. Total proteins were extracted from lung tissues or cells. Lung or cultured fibroblasts were lysed with radioimmunoprecipitation assay (RIPA) buffer (1\% NP-40, $0.5 \%$ sodium deoxycholate, $0.1 \%$ SDS, $150 \mathrm{mM}$ $\mathrm{NaCl}, 1 \mathrm{mM}$ EDTA, and $50 \mathrm{mM}$ Tris- $\mathrm{HCl}, \mathrm{pH} 7.5)$ and centrifuged at $14,000 \mathrm{x}$ g for $10 \mathrm{~min}$ at $4^{\circ} \mathrm{C}$. Protein concentrations in supernatants were measured with a Bradford assay (Beijing Solarbio Science \& Technology Co., Ltd., Beijing, China). Protein samples (20 $\mu \mathrm{g} / \mathrm{lane})$ were separated by $10 \%$ SDS-PAGE and transferred onto polyvinylidene fluoride membranes. Membranes were blocked with 5\% non-fat milk for $2 \mathrm{~h}$ at room temperature and incubated overnight at $4^{\circ} \mathrm{C}$ with the primary antibody against $\mathrm{Col} \mathrm{I}$, $\alpha$-SMA, Fn, ACE and AT1 (all 1:500) followed by a peroxidase-labeled affinity-purified secondary antibody to rabbit/mouse IgG (1:5,000, cat. no. 074-1506, Kirkegaard \& Perry Laboratories, Inc., Gaithersburg, MD, USA) for $2 \mathrm{~h}$ at $37^{\circ} \mathrm{C}$. Target bands were visualized by the addition of an Enhanced Chemiluminescence Prime Western Blotting Detection reagent (GE Healthcare, Chicago, IL, USA). The density of specific bands was analyzed using Image-Pro Plus image processing software (version 6.0; Media Cybernetics, Inc., Rockville, MD, USA) and the results were normalized to $\beta$-actin or GAPDH (both 1:500).

ELISA assay. Lung tissues were lysed with RIPA and the levels of Ac-SDKP and Ang II were determined by using the corresponding ELISA kits according to the manufacturer's protocol.

Statistical analysis. All experiments were performed in triplicate. The results are expressed as the mean \pm standard error of the mean. Comparisons between multiple independent groups were performed using one-way analysis of variance followed by a post hoc analysis with the Bonferroni test. $\mathrm{P}<0.05$ was considered to indicate a statistically significant difference.

\section{Results}

Morphological alterations in lungs of rats exposed to silica. The results of HE staining revealed that in the normal controls, the alveolar walls were thin and there was no obvious inflammatory cell infiltration (Fig. 1A). In the 2-week silicosis group, macrophages were observed in the alveoli and the alveolar wall was wider in appearance. The 4-week silicosis group exhibited isolated cell nodules that contained macrophages. The 8- and 12-week silicosis groups had significantly wider alveolar walls and a greater number of silicotic nodules. The 16-week silicosis group exhibited an increase in silicotic nodule volume, nodule fusion, interstitial fibrosis and cell fibrous nodules (Fig. 1A). Blue purple Masson trichrome staining was observed in the nodules and interstitial fibrosis area, indicating collagen deposition, which gradually increased with model preparation time and most distinct in the 16-week silicosis group (Fig. 1B).

Expression of ACE, Ang II, AT1 and Ac-SDKP in the lungs of rats exposed to silica. To investigate whether ACE/Ang II/AT1 proteins are involved in the pathogenesis of silicotic fibrosis, the expression of ACE, Ang II and AT1 in rat lungs was assessed. The expression of ACE, Ang II and AT1 was increased in the 2-, 4-, 8-, 12- and 16-week silicosis groups compared with the control group (Fig. 2A and B). Ac-SDKP expression in the 2-week silicosis group was significantly higher compared with the control group, whereas expression in the 4-, 8-, 12- and 16-week silicosis groups decreased (Fig. 2C).

Expression of $\alpha$-SMA, Col I and Fn in silicosis and in fibroblasts induced by Ang II. Western blotting revealed that, compared with the control group, $\alpha$-SMA, Col I and Fn expression was gradually increased in the 4-, 8-, 12- and 16-week silicosis groups (Fig. 3A). In response to Ang II stimuli, the cultured rat lung fibroblasts underwent differentiation and produced excessive amounts of ECM in vitro. Ang II stimulated the expression of $\alpha$-SMA, Col I and Fn, which increased with treatment duration (Fig. 3B). Based on these findings and results of a previous study (19), $24 \mathrm{~h}$ was selected as the optimal treatment time for subsequent studies.

Ac-SDKP regulates ACE, Ang II and ATl during silicosis and the expression of AT1 in fibroblasts induced by Ang II. Compared with the 16-week silicosis group, treatment with 

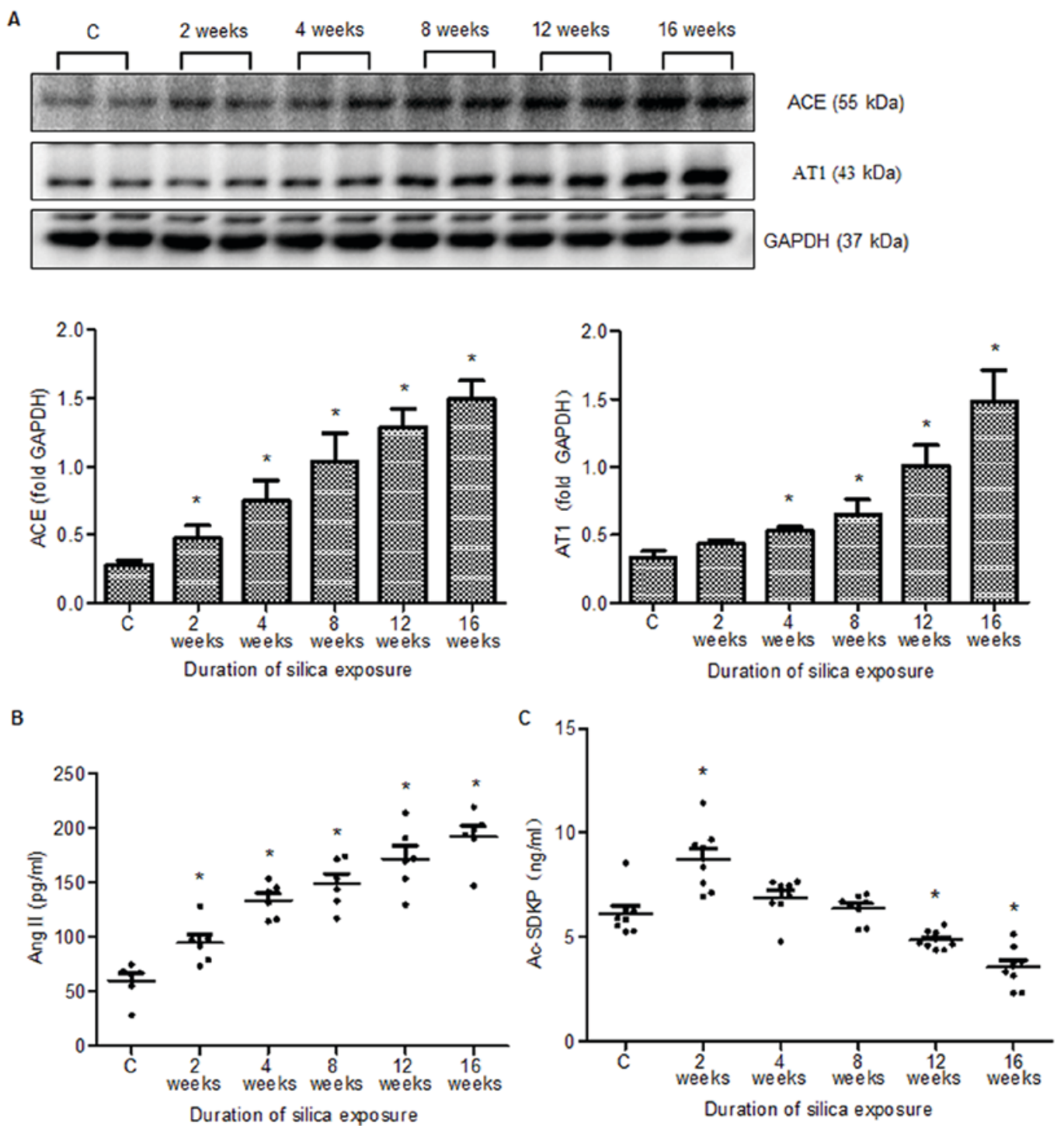

Figure 2. Expression of ACE, Ang II, AT1 and Ac-SDKP in rats. (A) ACE and AT1 expression in silicotic rat lung tissues were assessed by western blotting. (B) Ang II and (C) Ac-SDKP expression in rat silicotic lung tissues was assessed using ELISA. " $\mathrm{P}<0.05$ vs. the control group. ACE, angiotensin-converting enzyme; Ang II, angiotensin II; Ac-SDKP, N-acetyl-seryl-aspartyl-lysyl-proline; AT1, angiotensin II type 1.

Ac-SDKP resulted in decreased AT1 expression. The expression of ACE and Ang II (Fig. 4A and B) in the Ac-SDKP treatment group decreased, but was not statistically significant. The Ang II-dependent increase in AT1 expression was also inhibited following Ac-SDKP and valsartan treatment (Fig. 4C).

Ac-SDKP attenuates the expression of $\alpha$-SMA, Col I and Fn during silicosis and in Ang II-induced fibroblasts. IHC staining revealed that $\alpha$-SMA-positive myofibroblasts were located in the nodules and distributed in the interstitial fibrotic area in the 16-week silicosis group (Fig. 5). Ac-SDKP treatment reduced the expression of $\alpha$-SMA. Compared with the 16-week silicosis group, $\alpha$-SMA, Col I and Fn expression levels were significantly downregulated in the Ac-SDKP post-treatment group (Fig. 6). Subsequently, $\alpha$-SMA expression was assessed using IHC staining. Compared with the control group, $\alpha$-SMA expression was demonstrated to be increased following Ang II induction in cultured lung fibroblasts. Compared with the Ang II group, the expression of $\alpha$-SMA was decreased in the Ac-SDKP and valsartan treatment groups (Fig. 7). The Ang II-dependent increase in $\alpha$-SMA, Col I and Fn expression was also inhibited following Ac-SDKP and valsartan treatment (Fig. 8).

Ac-SDKP regulates MMP-1, TIMP-1 and the MMP-1/TIMP-1 ratio during silicosis and in fibroblasts induced by Ang II. Western blotting demonstrated that the expression of TIMP-1 was markedly increased in the silicosis group compared with the control group, whereas MMP-1 expression and the MMP-1/TIMP-1 ratio was significantly decreased (Fig. 9A). Treatment with Ac-SDKP increased the expression of MMP-1 and the MMP-1/TIMP-1 ratio. No significant differences in TIMP-1 expression were observed between the Ac-SDKP post-treatment and silicosis groups. In vitro experiments revealed that MMP-1 expression and the MMP-1/TIMP-1 ratio was reduced compared with the control and the expression of TIMP-1 was markedly increased in fibroblasts induced by Ang II (Fig. 9B). Ac-SDKP or valsartan treatment upregulated the expression of MMP-1 and enhanced the MMP-1/TIMP-1 

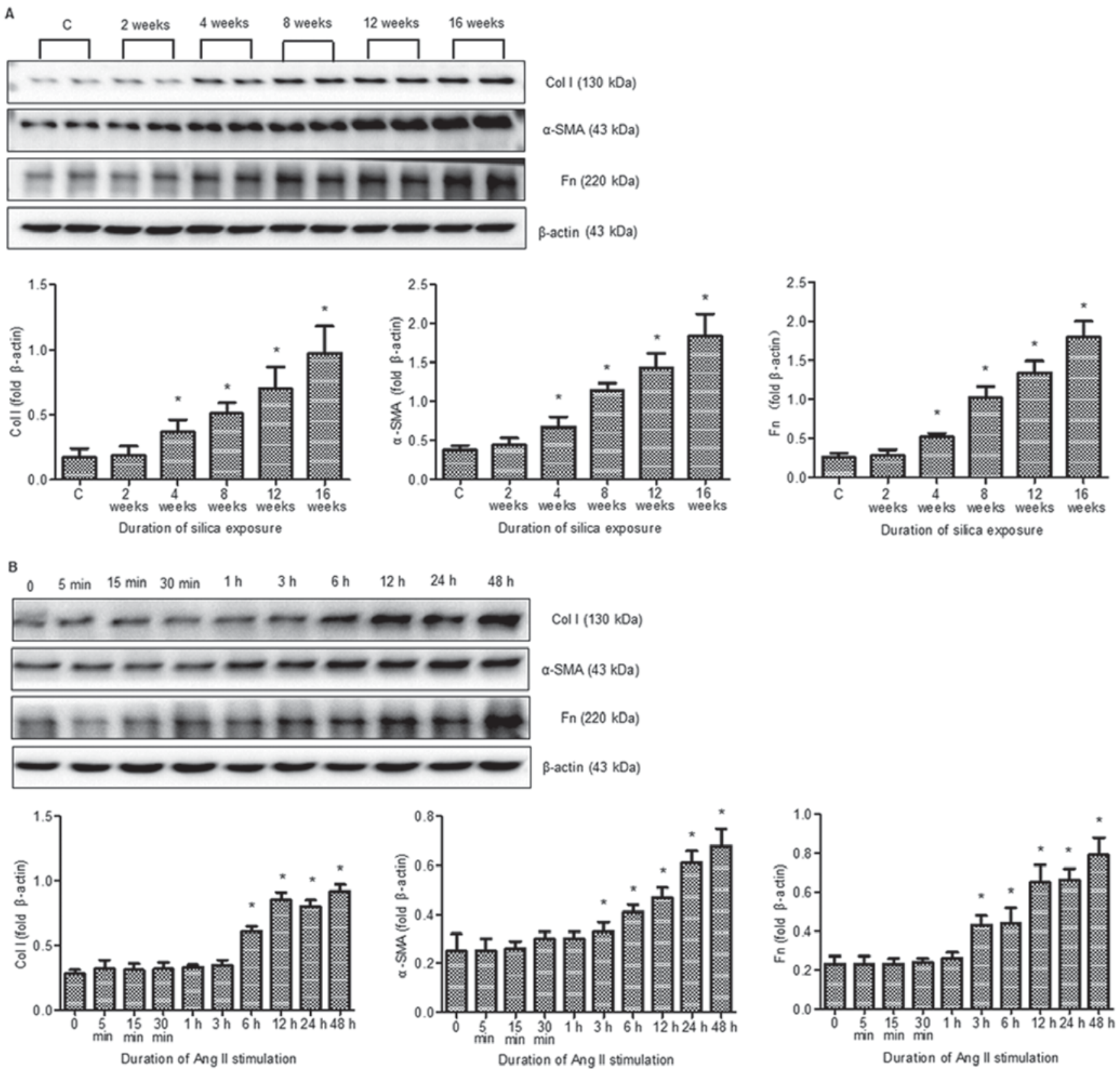

Figure 3. Expression of $\alpha$-SMA, Col I and Fn in silicosis and in Ang-II stimulated fibroblasts. $\alpha$-SMA, Col I and Fn expression in (A) lung tissues and (B) fibroblasts at different time points was assessed using western blotting. ${ }^{*} \mathrm{P}<0.05$ vs. the control group. $\alpha$-SMA, $\alpha$-smooth muscle actin; Col I, collagen I; Fn, Fibronectin; Ang II, angiotensin II; C, control.

ratio, whereas it had no significant effect on the expression of TIMP-1.

\section{Discussion}

In the present study, a rat silicosis model was established using HOPE MED8050 exposure control apparatus. This system is a non-invasive instrument that allows animal inhalation of silica particles and may be set to a certain dust concentration. Compared with intratracheal administration of silica dust $(20,21)$, using the dynamic pollution control system facilitated the establishment of an ideal silicosis model. Histopathological observations demonstrated that, following inhalation of $\mathrm{SiO}_{2}$ for 4 weeks, silicotic nodules containing macrophages developed in the lung tissues of rats. Furthermore, the number of silicotic nodules increased after 8 and 12 weeks of inhalation. Fibrous and cellular silicotic nodules with diffuse interstitial fibrosis were observed in rats at 16 weeks. Myofibroblasts are $\alpha$-SMA-expressing cells that originate from epithelial-mesenchymal transition (22), endothelial-to-mesenchymal transition (23), pericyte transdifferentiation (24) and pleural mesothelial cells (25) or pulmonary interstitial fibroblasts (26). Myofibroblasts serve a pivotal role in wound healing; however, the unusual persistent proliferative and migratory properties of myofibroblasts leads to an excessive accumulation of the ECM and lung fibrosis. In the present study, IHC revealed that $\alpha$-SMA-positive myofibroblasts surrounded macrophages and were irregularly 

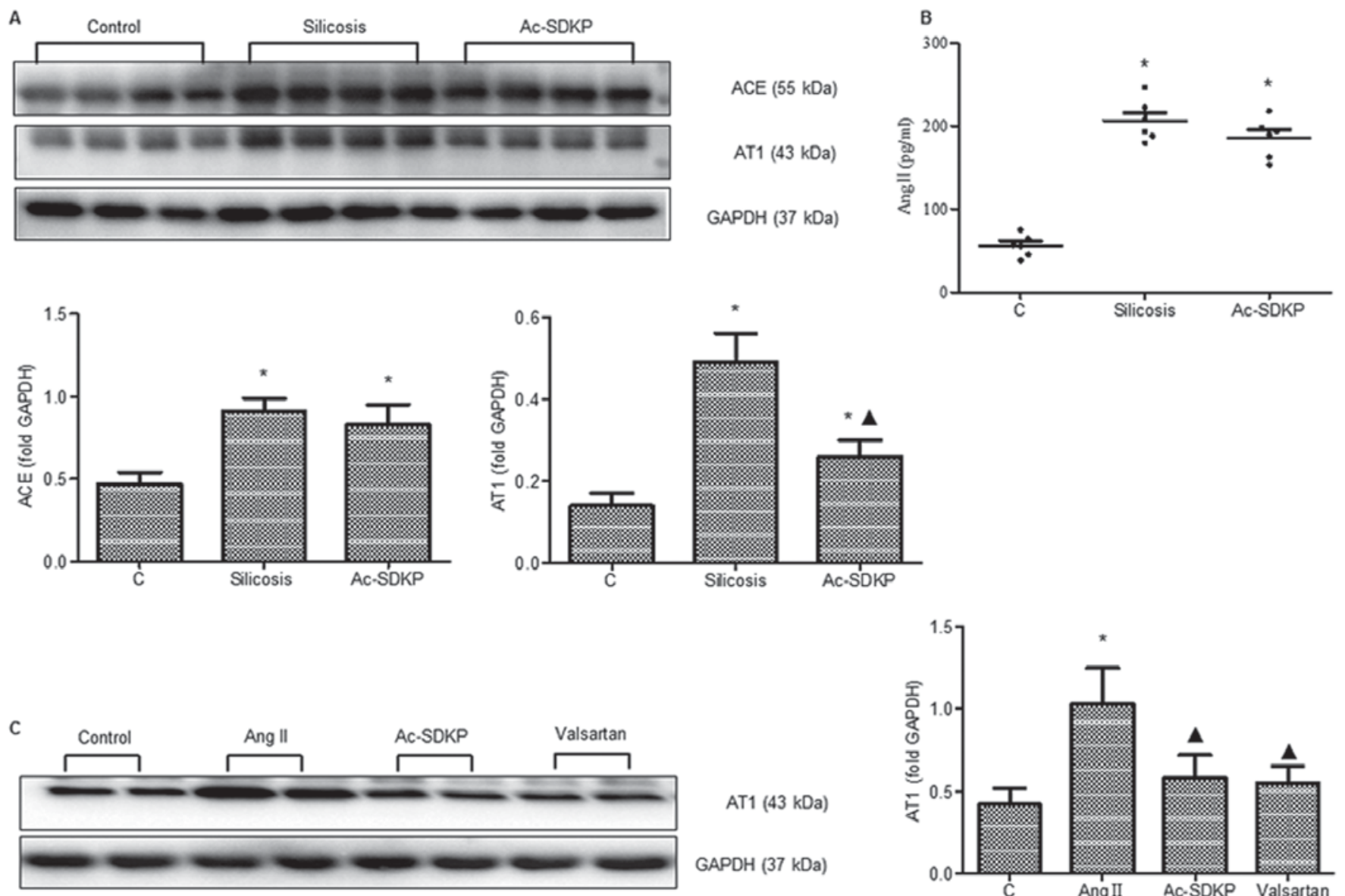

AT1 (43 kDa)

GAPDH $(37 \mathrm{kDa})$

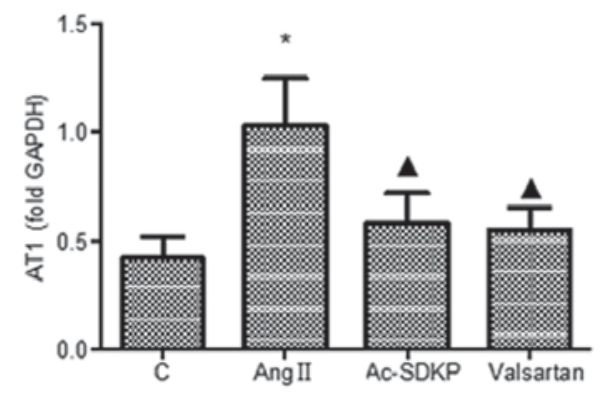

Figure 4. Ac-SDKP regulates ACE, Ang II and AT1 contents in silicosis and the levels of AT1 in Ang II-induced fibroblasts. (A) ACE and AT1 expression in silicotic rat lung tissues was assessed using western blotting. ${ }^{*} \mathrm{P}<0.05$ vs. the control group; ${ }^{\wedge} \mathrm{P}<0.05$, vs. the silicosis group. (B) Ang II levels in rat silicotic lung tissues were assessed using ELISA. " $\mathrm{P}<0.05$ vs. the control group. (C) AT1 expression in fibroblasts was assessed using western blotting. "P<0.05 vs. the control group; ${ }^{\wedge} \mathrm{P}<0.05$, compared with Ang II group. Ac-SDKP, N-acetyl-seryl-aspartyl-lysyl-proline; Ang II, angiotensin II; AT1, angiotensin II type 1; ACE, angiotensin-converting enzyme; $\mathrm{C}$, control.
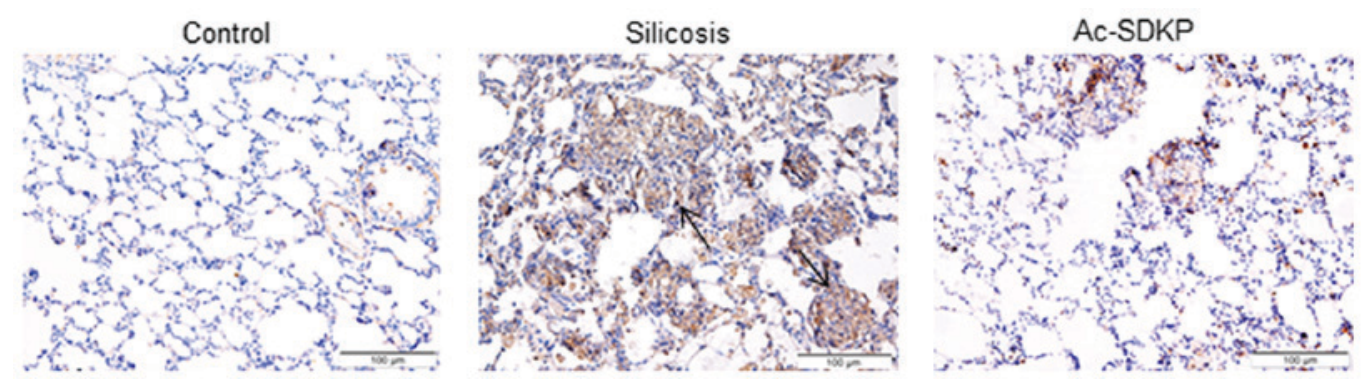

Figure 5. Immunohistochemistry measurement of $\alpha$-smooth muscle actin expression in lung tissues. The arrows refer to nodules and interstitial fibrotic area. Scale bars, $100 \mu \mathrm{m}$. Ac-SDKP, N-acetyl-seryl-aspartyl-lysyl-proline.

distributed in interstitial fibrotic areas. Western blotting further demonstrated that $\alpha$-SMA protein expression in the 4-, 8-, 12- and 16-week silicosis groups gradually increased, suggesting the accumulation of a large number of activated myofibroblasts in the silicosis model. These findings suggested that a silicosis model was successfully established using a HOPE-MED8050 dynamic dust system.

RAS is a key mediator that is involved in the pathogenesis of tissue remodeling, including the lungs (5). Studies have reported that the expression of ACE in bronchoalveolar lavage fluid is higher (27) and the angiotensinogen gene is overexpressed in the lung tissues of patients with pulmonary fibrosis (28). This contributes to increase Ang II levels in pulmonary fibrosis. It has been suggested that Ang II, acting via the AT1 receptor, modulates pro-fibrotic downstream effects, including inflammatory cell recruitment, cellular proliferation and the accumulation of ECM (8). In the present study it was demonstrated that the expression of ACE, Ang II and AT1 in the lung tissue of rats increased with time, which was consistent with the degree of silicosis. Differentiation of fibroblasts into myofibroblasts is a critical event in fibrosis. Ang II serves a major role in fibrosis by promoting myofibroblast 

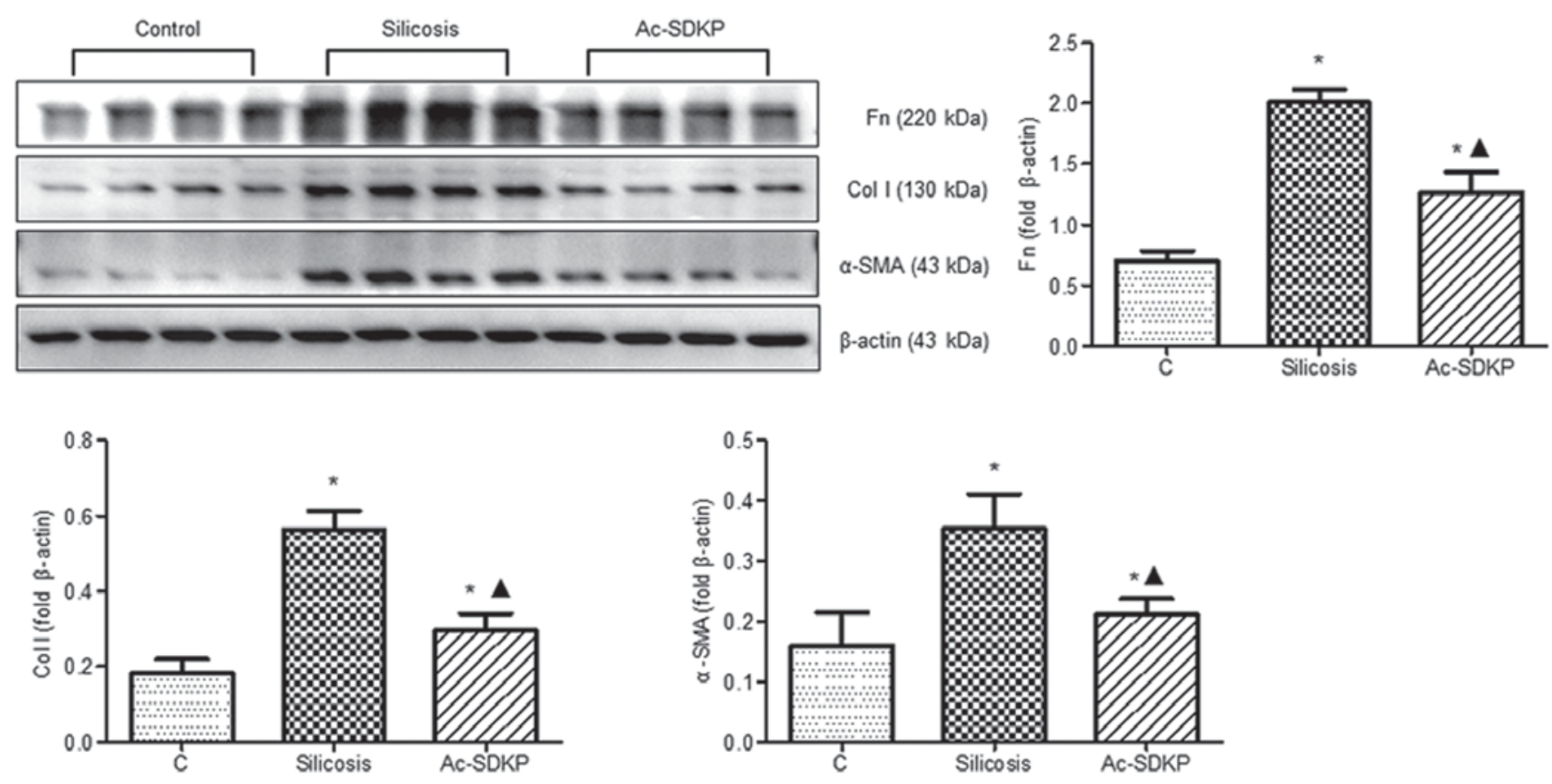

Figure 6. Ac-SDKP attenuates the expression of $\alpha$-SMA, Col I and Fn in silicosis. $\alpha$-SMA, Col I and Fn expression in lung tissues was assessed using western blotting. ${ }^{*} \mathrm{P}<0.05$ vs. the control group; ${ }^{\wedge} \mathrm{P}<0.05$, vs. the silicosis group. Ac-SDKP, N-acetyl-seryl-aspartyl-lysyl-proline; $\alpha$-SMA, $\alpha$-smooth muscle actin; Fn, Fibronectin; C, control.
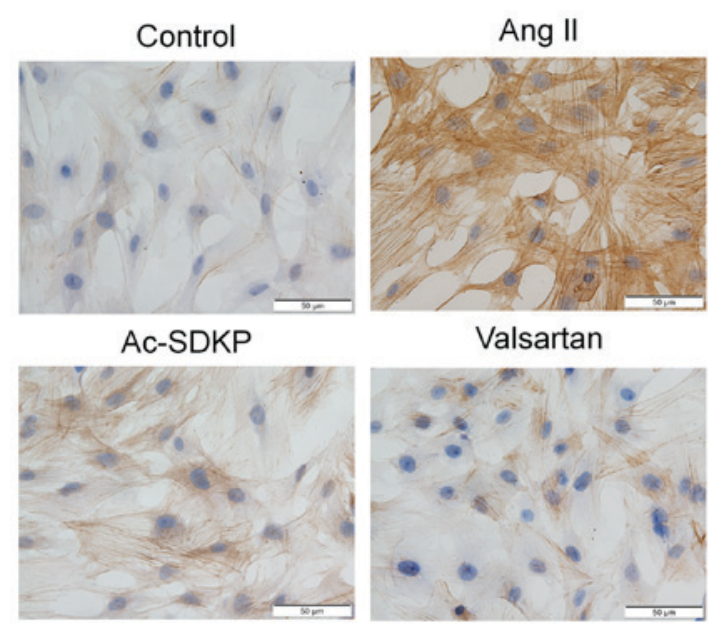

Figure 7.Immunohistochemistry measurement of $\alpha$-SMA expression in Ang IIstimulated fibroblasts. Scale bar, $50 \mu \mathrm{m}$. Ang II, angiotensin II; $\alpha$-SMA, $\alpha$-smooth muscle actin; Ac-SDKP, N-acetyl-seryl-aspartyl-lysyl-proline.

differentiation (29,30). The expression of $\alpha$-SMA, Col I and Fn in Ang II-stimulated lung fibroblasts was demonstrated to be time-dependent in the present study, thereby confirming that myofibroblast differentiation may be induced by Ang II from lung fibroblasts and suggesting that the increased ECM deposition in the rat silicosis model may be associated with Ang II elevation in vivo. Silicosis in the rat model was mediated, at least partially, by activation of the RAS signaling pathway.

Ac-SDKP is an ACE substrate that demonstrates meaningful anti-fibrotic effects in various experimental models of fibrotic disease (3). Ac-SDKP has been reported to inhibit cell proliferation and myofibroblast differentiation via weakening TGF- $\beta$-induced mothers against decapentaplegic homolog 2 (Smad2)/3 signaling effects (31-33). It has also been demonstrated that Ac-SDKP treatment promotes the expression of Smad7, which antagonizes Smad2 activity (34). Both RAS and TGF- $\beta$ have been described as important regulators of the fibrotic disease $(35,36)$. Ang II and TGF- $\beta 1$ do not appear to act independently from one another, but rather as part of a signaling network in cardiac remodeling and fibrosis $(14,37)$. Therefore, the interaction between Ac-SDKP and RAS in fibrosis requires further investigation. In the present study, it was observed that Ac-SDKP levels in lung tissues were increased in the 2-week silicosis group compared with the control group, whereas the expression of Ac-SDKP decreased gradually at subsequent time points. Decreased Ac-SDKP expression and increased ACE, Ang II and AT1 expression may result in an imbalance between pro-fibrotic and anti-fibrotic molecules, thereby facilitating the occurrence and development of silicosis. In addition, the results of the present study revealed that Ac-SDKP treatment did not significantly affect ACE or Ang II levels, whereas Ac-SDKP did downregulate the expression of AT1 in vivo and inhibited myofibroblast differentiation and ECM synthesis. Ac-SDKP had the same effect as valsatan, inhibiting Ang II-induced myofibroblast differentiation and decreasing Col I and Fn accumulation in vitro. The fibrogenic process is associated with an imbalance in MMPs/TIMPs, which results in excessive expression of components of the ECM. MMP-1 serves an important role in fibrosis by preferentially degrading Col I (38). In contrast, TIMP-1 moderately inhibits the activity of MMP1 (39). In the present study, Ac-SDKP treatment increased the expression levels MMP-1 and the MMP-1/TIMP-1 ratio in vivo and in vitro. The results of the present study therefore suggest that Ac-SDKP may effectively protect lung tissues against silicosis fibrosis and serve a vital role in inhibiting the Ang II-induced myofibroblast differentiation and accumulation of ECM in silicosis. 

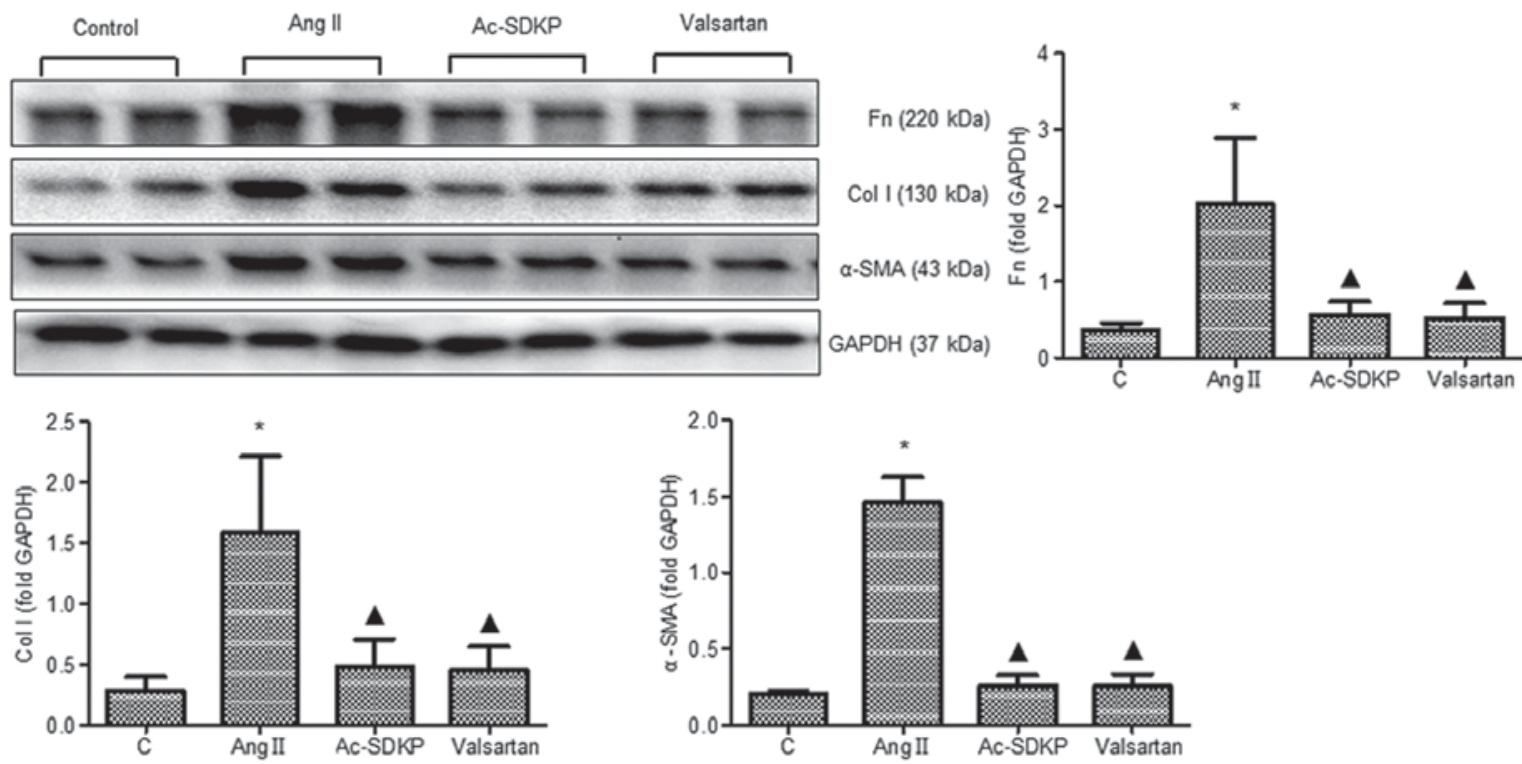

Figure 8. Ac-SDKP attenuates the expression of $\alpha$-SMA, Col I and Fn in Ang II-stimulated fibroblasts. $\alpha$-SMA, Col I and Fn expression in fibroblasts were assessed using western blotting. "P $<0.05$ vs. the control group; ${ }^{\wedge} \mathrm{P}<0.05$, compared with Ang II group. Ac-SDKP, N-acetyl-seryl-aspartyl-lysyl-proline; $\alpha$-SMA, $\alpha$-smooth muscle actin; Fn, Fibronectin; Col I, collagen I; C, control.
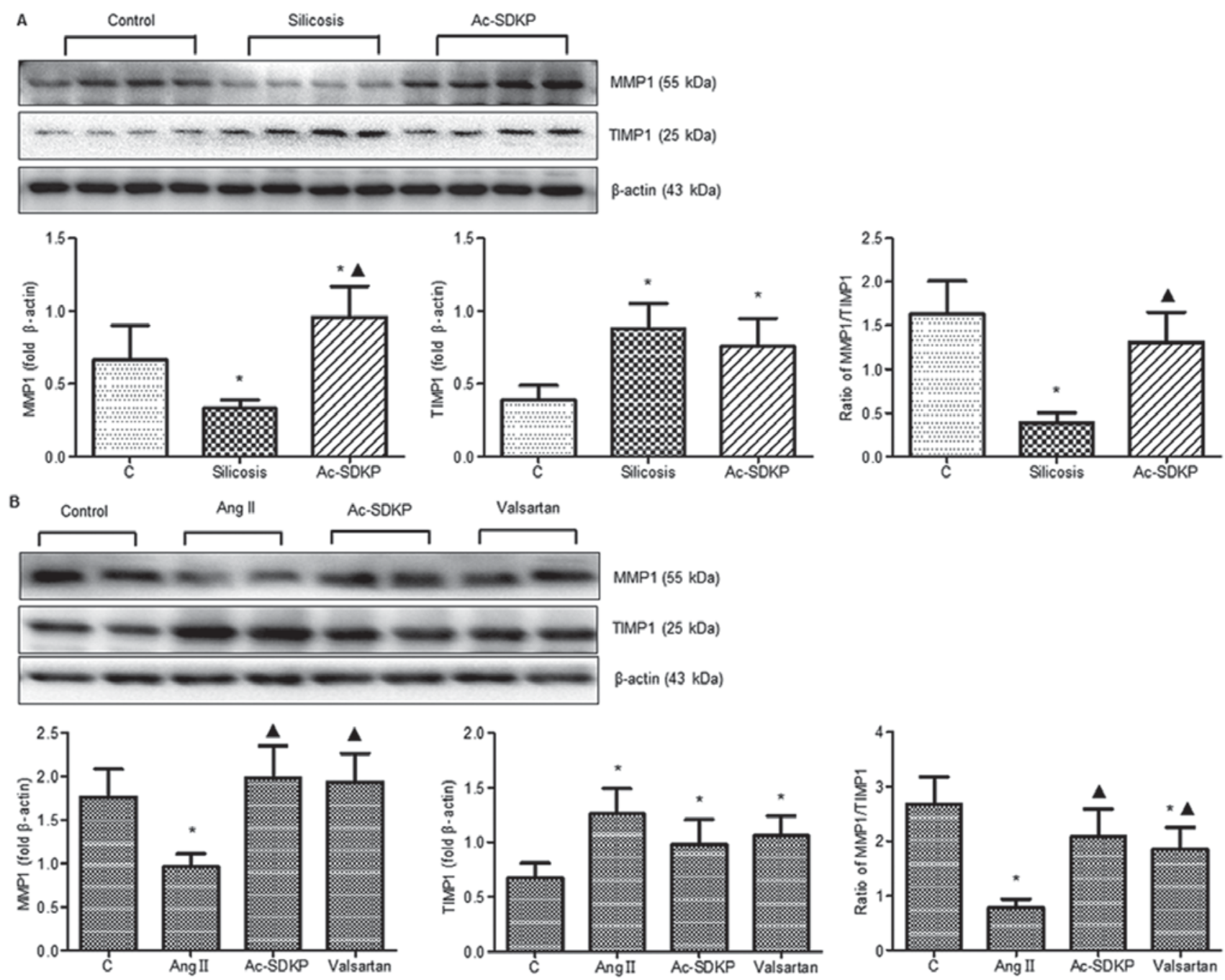

Figure 9. Ac-SDKP regulates the expression of MMP-1, TIMP-1 and the MMP-1/TIMP-1 ratio in silicosis and in Ang II-stimulated fibroblasts. MMP-1 and TIMP-1 expression and the MMP-1/TIMP-1 ratio were assessed in (A) lung tissues and (B) fibroblasts using western blotting. "P<0.05 vs. the control group; $\triangle \mathrm{P}<0.05$, compared with Ang II group. Ac-SDKP, N-acetyl-seryl-aspartyl-lysyl-proline; MMP-1, matrix metalloproteinase-1; TIMP-1, tissue inhibitor of metalloproteinases-1; C, control. 
In summary, the results of the present study suggest that an imbalance between Ac-SDKP and ACE/Ang II/AT1 molecules promotes the development of silicosis. Ac-SDKP blocked fibroblast differentiation induced by Ang II and inhibited ECM production, thereby preventing the development of silicotic fibrosis. As Ac-SDKP is inhibited by ACE in vivo, the authors of the present study intend to use an ACE inhibitor to determine if similar anti-fibrosis results can be observed. The present study indicates a possibility of use of Ac-SDKP as an inhibitor of RAS for the treatment of silicosis.

\section{Acknowledgements}

The present study was supported by the National Natural Science Foundation of China (grant no. 81472953), the Natural Science Foundation of Hebei Province (grant no. H2016209170), the National College Students Innovation and Entrepreneurship Training Program (grant no. 201710081010) and Doctoral Fund of North China University of Science and Technology.

\section{References}

1. Rimal B, Greenberg AK and Rom WN: Basic pathogenetic mechanisms in silicosis: Current understanding. Curr Opin Pulm Med 11: 169-173, 2005.

2. Cavasin MA, Rhaleb NE, Yang XP and Carretero OA: Prolyl oligopeptidase is involved in release of the antifibrotic peptide Ac-SDKP. Hypertension 43: 1140-1145, 2004.

3. Mnguni AT, Engel ME, Borkum MS and Mayosi BM: The effects of angiotensin converting enzyme inhibitors (ACE-I) on human $\mathrm{N}$-acetyl-seryl-aspartyl-lysyl-proline (Ac-SDKP) levels: A systematic review and meta-analysis. PLoS One 10: e0143338, 2015.

4. Kumar N, Nakagawa P, Janic B, Romero CA, Worou ME, Monu SR, Peterson EL, Shaw J, Valeriote F, Ongeri EM, et al: The anti-inflammatory peptide Ac-SDKP is released from thymosin- $\beta 4$ by renal meprin- $\alpha$ and prolyl oligopeptidase. Am J Physiol Renal Physiol 310: F1026-F1034, 2016.

5. Meng Y, Yu CH, Li W, Li T, Luo W, Huang S, Wu PS, Cai SX and Li X: Angiotensin-converting enzyme 2/angiotensin-(1-7)/Mas axis protects against lung fibrosis by inhibiting the MAPK/NF- $\kappa \mathrm{B}$ pathway. Am J Respir Cell Mol Biol 50: 723-736, 2014.

6. Ntsekhe M, Matthews K, Wolske J, Badri M, Wilkinson KA, Wilkinson RJ, Sturrock ED and Mayosi BM: Scientific letter: Ac-SDKP (N-acetyl-seryl-aspartyl-lysyl-proline) and Galectin-3 levels in tuberculous pericardial effusion: Implications for pathogenesis and prevention of pericardial constriction. Heart 98: 1326-1328, 2012.

7. Wang J, Chen L, Chen B, Meliton A, Liu SQ, Shi Y,Liu T, Deb DK, Solway J and Li YC: Chronic activation of the renin-angiotensin system induces lung fibrosis. Sci Rep 5: 15561, 2015.

8. Murphy AM, Wong AL and Bezuhly M: Modulation of angiotensin II signaling in the prevention of fibrosis. Fibrogenesis Tissue Repair 8: 7, 2015.

9. Wei L, Alhenc-Gelas F, Corvol P and Clauser E: The two homologous domains of human angiotensin I-converting enzyme are both catalytically active. J Biol Chem 266: 9002-9008, 1991.

10. Masuyer G, Schwager SL, Sturrock ED, Isaac RE and Acharya KR: Molecular recognition and regulation of human angiotensin-I converting enzyme (ACE) activity by natural inhibitory peptides. Sci Rep 2: 717, 2012.

11. Stawski L, Han R, Bujor AM and Trojanowska M: Angiotensin II induces skin fibrosis: A novel mouse model of dermal fibrosis. Arthritis Res Ther 14: R194, 2012

12. Cavin S, Maric D and Diviani D: A-kinase anchoring protein-Lbc promotes pro-fibrotic signaling in cardiac fibroblasts. Biochim Biophys Acta 1843: 335-345, 2014

13. Wong MH,Chapin OC and Johnson MD: LPS-stimulated cy tokine production in type I cells is modulated by the renin-angiotensin system. Am J Respir Cell Mol Biol 46: 641-650, 2012.

14. Uhal BD, Kim JK, Li X and Molina-Molina M: AngiotensinTGF-beta 1 crosstalk in human idiopathic pulmonary fibrosis: Autocrine mechanisms in myofibroblasts and macrophages. Curr Pharm Des 13: 1247-1256, 2007.
15. Masuyer G, Douglas RG, Sturrock ED and Acharya KR: Structural basis of Ac-SDKP hydrolysis by Angiotensin-I converting enzyme. Sci Rep 5: 13742, 2015.

16. Liu Y, Xu H, Geng Y, Xu D, Zhang L, Yang Y, Wei Z, Zhang B, Li S, Gao X, et al: Dibutyryl-cAMP attenuates pulmonary fibrosis by blocking myofibroblast differentiation via PKA/CREB/CBP signaling in rats with silicosis. Respir Res 18: 38, 2017.

17. Xu H, Yang F, Sun Y, Yuan Y, Cheng H, Wei Z, Li S, Cheng T, Brann D and Wang R: A new antifibrotic target of Ac-SDKP: Inhibition of myofibroblast differentiation in rat lung with silicosis. PLoS One 7: e40301, 2012.

18. Li S, Gao X, Xu D, Wang X, Liu Y, Zhang L, Deng H, Wei Z, Tian J, Xu H and Yang F: Inhibition effect of N-acetyl-seryl-a spartyl-lysyl-proline on myofibroblast differentiation by regulating acetylated tubulin $\alpha$ in silicotic rat model. Zhonghua Lao Dong Wei Sheng Zhi Ye Bing Za Zhi 33: 816-821, 2015 (In Chinese).

19. Meng Y, Li T, Zhou GS, Chen Y, Yu CH, Pang MX, Li W, $\mathrm{Li}$ Y, Zhang WY and Li X: The angiotensin-converting enzyme 2/angiotensin (1-7)/Mas axis protects against lung fibroblast migration and lung fibrosis by inhibiting the NOX4-derived ROS-mediated RhoA/Rho kinase pathway. Antioxid Redox Signal 22: 241-258, 2015.

20. Morimoto Y, Nagatomo H, Hirohashi M, Oyabu T, Ogami A, Yamato H, Kuroda K, Obata Y, Higashi T and Tanaka I: Expression of clara cell secretory protein in the lungs of rats exposed to crystalline silica in vivo. J Occup Health 47: 504-509, 2005.

21. Dong H, Jing W, Yabo Y, Xiaokang Y, Wan W, Min M, Wenyang W, Zhaoquan C, Yingru X and Rongbo Z: Establishment of rat model of silicotuberculosis and its pathological characteristic. Pathog Glob Health 108: 312-316, 2014.

22. Zhou B, Liu Y, Kahn M, Ann DK, Han A, Wang H, Nguyen C, Flodby P, Zhong Q, Krishnaveni MS, et al: Interactions between $\beta$-catenin and transforming growth factor- $\beta$ signaling pathways mediate epithelial-mesenchymal transition and are dependent on the transcriptional co-activator cAMP-response element-binding protein (CREB)-binding protein (CBP). J Biol Chem 287: 7026-7038, 2012.

23. Hashimoto N, Phan SH, Imaizumi K, Matsuo M, Nakashima H, Kawabe T, Shimokata K and Hasegawa Y: Endothelialmesenchymal transition in bleomycin-induced pulmonary fibrosis. Am J Respir Cell Mol Biol 43: 161-172, 2010.

24. Marriott S, Baskir RS, Gaskill C, Menon S, Carrier EJ, Williams J, Talati M, Helm K, Alford CE, Kropski JA, et al: ABCG2pos lung mesenchymal stem cells are a novel pericyte subpopulation that contributes to fibrotic remodeling. Am J Physiol Cell Physiol 307: C684-C698, 2014.

25. Zolak JS, Jagirdar R, Surolia R, Karki S, Oliva O, Hock T, Guroji P, Ding Q, Liu RM, Bolisetty S, et al: Pleural mesothelial cell differentiation and invasion in fibrogenic lung injury. Am J Pathol 182: 1239-1247, 2013.

26. Tumelty KE, Smith BD, Nugent MA and Layne MD: Aortic carboxypeptidase-like protein (ACLP) enhances lung myofibroblast differentiation through transforming growth factor $\beta$ receptor-dependent and -independent pathways. J Biol Chem 289: 2526-2536, 2014

27. Specks U, Martin WJ II and Rohrbach MS: Bronchoalveolar lavage fluid angiotensin-converting enzyme in interstitial lung diseases. Am Rev Respir Dis 141: 117-123, 1990.

28. Li X, Molina-Molina M, Abdul-Hafez A, Ramirez J, SerranoMollar A, Xaubet A and Uhal BD: Extravascular sources of lung angiotensin peptide synthesis in idiopathic pulmonary fibrosis. Am J Physiol Lung Cell Mol Physiol 291: L887-L895, 2006.

29. Bai J, Zhang N, Hua Y, Wang B, Ling L, Ferro A and Xu B: Metformin inhibits angiotensin II-induced differentiation of cardiac fibroblasts into myofibroblasts. PLoS One 8: e72120, 2013.

30. Guo X, Yan F, Li J, Zhang C and Bu P: SIRT3 attenuates AngII-induced cardiac fibrosis by inhibiting myofibroblasts transdifferentiation via STAT3-NFATc2 pathway. Am J Transl Res 9: 3258-3269, 2017.

31. Peng H, Carretero OA, Peterson EL and Rhaleb NE: Ac-SDKP inhibits transforming growth factor-beta1-induced differentiation of human cardiac fibroblasts into myofibroblasts. Am J Physiol Heart Circ Physiol 298: H1357-H1364, 2010.

32. Pokharel S, Rasoul S, Roks AJ, van Leeuwen RE, van Luyn MJ, Deelman LE, Smits JF, Carretero O, van Gilst WH and Pinto YM: $\mathrm{N}$-acetyl-Ser-Asp-Lys-Pro inhibits phosphorylation of Smad2 in cardiac fibroblasts. Hypertension 40: 155-161, 2002. 
33. Kanasaki K, Koya D, Sugimoto T, Isono M, Kashiwagi A and Haneda M: N-Acetyl-seryl-aspartyl-lysyl-proline inhibits TGF-beta-mediated plasminogen activator inhibitor-1 expression via inhibition of Smad pathway in human mesangial cells. J Am Soc Nephrol 14: 863-872, 2003.

34. Lin CX, Rhaleb NE, Yang XP, Liao TD, D'Ambrosio MA and Carretero OA: Prevention of aortic fibrosis by N-acetyl-seryl-asp artyl-lysyl-proline in angiotensin II-induced hypertension. Am J Physiol Heart Circ Physiol 295: H1253-H1261, 2008.

35. Yamazaki KG, Gonzalez E and Zambon AC: Crosstalk between the renin-angiotensin system and the advance glycation end product axis in the heart: Role of the cardiac fibroblast. J Cardiovasc Transl Res 5: 805-813, 2012.

36. Samarakoon R, Overstreet JM and Higgins PJ: TGF- $\beta$ signaling in tissue fibrosis: Redox controls, target genes and therapeutic opportunities. Cell Signal 25: 264-268, 2013.
37. Rosenkranz S: TGF-beta1 and angiotensin networking in cardiac remodeling. Cardiovase Res 63: 423-432, 2004.

38. Craig VJ, Zhang L, Hagood JS and Owen CA: Matrix metalloproteinases as therapeutic targets for idiopathic pulmonary fibrosis. Am J Respir Cell Mol Biol 53: 585-600, 2015.

39. Yoshiji H, Kuriyama S, Yoshii J, Ikenaka Y, Noguchi R, Nakatani T, Tsujinoue H, Yanase K, Namisaki T, Imazu H and Fukui H: Tissue inhibitor of metalloproteinases-1 attenuates spontaneous liver fibrosis resolution in the transgenic mouse. Hepatology 36: 850-860, 2002.

This work is licensed under a Creative Commons Attribution-NonCommercial-NoDerivatives 4.0 International (CC BY-NC-ND 4.0) License. 